\title{
Global Nonexistence of Positive Initial-Energy Solutions for Coupled Nonlinear Wave Equations with Damping and Source Terms
}

\author{
Liang Fei ${ }^{1,2}$ and Gao Hongjun ${ }^{1}$ \\ ${ }^{1}$ Jiangsu Provincial Key Laboratory for NSLSCS, School of Mathematical Science, \\ Nanjing Normal University, Nanjing 210046, China \\ ${ }^{2}$ Department of Mathematics, Anhui Science and Technology University, \\ Fengyang 233100, Anhui, China
}

Correspondence should be addressed to Liang Fei, fliangmath@126.com

Received 26 November 2010; Revised 5 June 2011; Accepted 27 June 2011

Academic Editor: Josef Diblík

Copyright (C) 2011 L. Fei and G. Hongjun. This is an open access article distributed under the Creative Commons Attribution License, which permits unrestricted use, distribution, and reproduction in any medium, provided the original work is properly cited.

This work is concerned with a system of nonlinear wave equations with nonlinear damping and source terms acting on both equations. We prove a global nonexistence theorem for certain solutions with positive initial energy.

\section{Introduction}

In this paper we study the initial-boundary-value problem

$$
\begin{gathered}
u_{t t}-\operatorname{div}\left(g\left(|\nabla u|^{2}\right) \nabla u\right)+\left|u_{t}\right|^{m-1} u_{t}=f_{1}(u, v), \quad(x, t) \in \Omega \times(0, T), \\
v_{t t}-\operatorname{div}\left(g\left(|\nabla v|^{2}\right) \nabla v\right)+\left|v_{t}\right|^{r-1} v_{t}=f_{2}(u, v), \quad(x, t) \in \Omega \times(0, T), \\
u(x, t)=v(x, t)=0, \quad x \in \partial \Omega \times(0, T), \\
u(x, 0)=u_{0}(x), \quad u_{t}(x, 0)=u_{1}(x), \quad x \in \Omega, \\
v(x, 0)=v_{0}(x), \quad v_{t}(x, 0)=v_{1}(x), \quad x \in \Omega,
\end{gathered}
$$

where $\Omega$ is a bounded domain in $\mathbb{R}^{n}$ with a smooth boundary $\partial \Omega, m, r \geq 1$, and $f_{i}(\cdot, \cdot): \mathbb{R}^{2} \rightarrow$ $\mathbb{R}(i=1,2)$ are given functions to be specified later. We assume that $g$ is a function which 
satisfies

$$
g \in C^{1}, \quad g(s)>0, \quad g(s)+2 s g^{\prime}(s)>0
$$

for $s>0$.

To motivate our work, let us recall some results regarding $g \equiv 1$. The single-wave equation of the form

$$
u_{t t}-\Delta u+h\left(u_{t}\right)=f(u), \quad x \in \Omega, t>0
$$

in $\Omega \times(0, \infty)$ with initial and boundary conditions has been extensively studied, and many results concerning global existence, blow-up, energy decay have been obtained. In the absence of the source term, that is, $(f=0)$, it is well known that the damping term $h\left(u_{t}\right)$ assures global existence and decay of the solution energy for arbitrary initial data (see [1]). In the absence of the damping term, the source term causes finite time blow-up of solutions with a large initial data (negative initial energy) (see $[2,3]$ ). The interaction between the damping term and the source term makes the problem more interesting. This situation was first considered by Levine $[4,5]$ in the linear damping case $h\left(u_{t}\right)=a u_{t}$ and a polynomial source term of the form $f(u)=b|u|^{p-2} u$. He showed that solutions with negative initial energy blow up in finite time. The main tool used in $[4,5]$ is the "concavity method." Georgiev and Todorova in [6] extended Levine's result to the nonlinear damping case $h\left(u_{t}\right)=a\left|u_{t}\right|^{m-2} u_{t}$. In their work, the authors considered problem (1.3) with $f(u)=b|u|^{p-2} u$ and introduced a method different from the one known as the concavity method and showed that solutions with negative energy continue to exist globally in time if $m \geq p \geq 2$ and blow up in finite time if $p>m \geq 2$ and the initial energy is sufficiently negative. This latter result has been pushed by Messaoudi [7] to the situation where the initial energy $E(0)<0$ and has been improved by the same author in [8] to accommodate certain solutions with positive initial energy.

In the case of $g$ being a given nonlinear function, the following equation:

$$
u_{t t}-g\left(u_{x}\right)_{x}-u_{x x t}+\delta\left|u_{t}\right|^{m-1} u_{t}=\mu|u|^{p-1} u, \quad x \in(0,1), t>0,
$$

with initial and boundary conditions has been extensively studied. Equation of type of (1.4) is a class of nonlinear evolution governing the motion of a viscoelastic solid composed of the material of the rate type, see [9-12]. It can also be seen as field equation governing the longitudinal motion of a viscoelastic bar obeying the nonlinear Voigt model, see [13]. In twoand three-dimensional cases, they describe antiplane shear motions of viscoelastic solids. We refer to [14-16] for physical origins and derivation of mathematical models of motions of viscoelastic media and only recall here that, in applications, the unknown $u$ naturally represents the displacement of the body relative to a fixed reference configuration. When $\delta=\mu=0$, there have been many impressive works on the global existence and other properties of solutions of (1.4), see [9, 10, 17, 18]. Especially, in [19] the authors have proved the global existence and uniqueness of the generalized and classical solution for the initial boundary value problem (1.4) when we replace $\delta\left|u_{t}\right|^{m-1} u_{t}$ and $\mu|u|^{p-1} u$ by $g\left(u_{t}\right)$ and $f(u)$, respectively. But about the blow-up of the solution for problem, in this paper there has not been any discussion. Chen et al. [20] considered problem (1.4) and first established an 
ordinary differential inequality, next given the sufficient conditions of blow-up of the solution of (1.4) by the inequality. In [21], Hao et al. considered the single-wave equation of the form

$$
u_{t t}-\operatorname{div}\left(g\left(|\nabla u|^{2}\right) \nabla u\right)+h\left(u_{t}\right)=f(u), \quad x \in \Omega, t>0
$$

with initial and Dirichlet boundary condition, where $g$ satisfies condition (1.2) and

$$
g(s) \geq b_{1}+b_{2} s^{q}, \quad q \geq 0 .
$$

The damping term has the form

$$
h\left(u_{t}\right)=d_{1} u_{t}+d_{2}\left|u_{t}\right|^{r-1} u_{t}, \quad r>1
$$

The source term is

$$
f(u)=a_{1} u+a_{2}|u|^{p-1} u
$$

with $p \geq 1$ for $n=1,2$ and $1 \leq n \leq 2 n /(n-2)$ for $n \geq 3, a_{1}, a_{2}, b_{1}, b_{2}, d_{1}, d_{2}$ are nonnegative constants, and $b_{1}+b_{2}>0$. By using the energy compensation method $[7,8,22]$, they proved that under some conditions on the initial value and the growth orders of the nonlinear strain term, the damping term, and the source term, the solution to problem (1.5) exists globally and blows up in finite time with negative initial energy, respectively.

Some special cases of system (1.1) arise in quantum field theory which describe the motion of charged mesons in an electromagnetic field, see [23, 24]. Recently, some of the ideas in $[6,22]$ have been extended to study certain systems of wave equations. Agre and Rammaha [25] studied the system of (1.1) with $g \equiv 1$ and proved several results concerning local and global existence of a weak solution and showed that any weak solution with negative initial energy blows up in finite time, using the same techniques as in [6]. This latter blow-up result has been improved by Said-Houari [26] by considering a larger class of initial data for which the initial energy can take positive values. Recently, Wu et al. [27] considered problem (1.1) with the nonlinear functions $f_{1}(u, v)$ and $f_{2}(u, v)$ satisfying appropriate conditions. They proved under some restrictions on the parameters and the initial data several results on global existence of a weak solution. They also showed that any weak solution with initial energy $E(0)<0$ blows up in finite time.

In this paper, we also consider problem (1.1) and improve the global nonexistence result obtained in [27], for a large class of initial data in which our initial energy can take positive values. The main tool of the proof is a technique introduced by Payne and Sattinger [28] and some estimates used firstly by Vitillaro [29], in order to study a class of a single-wave equation. 


\section{Preliminaries and Main Result}

First, let us introduce some notation used throughout this paper. We denote by $\|\cdot\|_{q}$ the $L^{q}(\Omega)$ norm for $1 \leq q \leq \infty$ and by $\|\nabla \cdot\|_{2}$ the Dirichlet norm in $H_{0}^{1}(\Omega)$ which is equivalent to the $H^{1}(\Omega)$ norm. Moreover, we set

$$
(\varphi, \psi)=\int_{\Omega} \varphi(x) \psi(x) d x
$$

as the usual $L^{2}(\Omega)$ inner product.

Concerning the functions $f_{1}(u, v)$ and $f_{2}(u, v)$, we take

$$
\begin{aligned}
& f_{1}(u, v)=\left[a|u+v|^{2(p+1)}(u+v)+b|u|^{p} u|v|^{(p+2)}\right], \\
& f_{2}(u, v)=\left[a|u+v|^{2(p+1)}(u+v)+b|u|^{(p+2)}|v|^{p} v\right],
\end{aligned}
$$

where $a, b>0$ are constants and $p$ satisfies

$$
\begin{cases}p>-1, & \text { if } n=1,2 \\ -1<p \leq \frac{4-n}{n-2}, & \text { if } n \geq 3 .\end{cases}
$$

One can easily verify that

$$
u f_{1}(u, v)+v f_{2}(u, v)=2(p+2) F(u, v), \quad \forall(u, v) \in \mathbb{R}^{2},
$$

where

$$
F(u, v)=\frac{1}{2(p+2)}\left[a|u+v|^{2(p+2)}+2 b|u v|^{p+2}\right]
$$

We have the following result.

Lemma 2.1 (see [30, Lemma 2.1]). There exist two positive constants $c_{0}$ and $c_{1}$ such that

$$
\frac{c_{0}}{2(p+2)}\left(|u|^{2(p+2)}+|v|^{2(p+2)}\right) \leq F(u, v) \leq \frac{c_{1}}{2(p+2)}\left(|u|^{2(p+2)}+|v|^{2(p+2)}\right) .
$$

Throughout this paper, we define $g$ by

$$
g(s)=b_{1}+b_{2} s^{q}, \quad q \geq 0, b_{1}+b_{2}>0,
$$

where $b_{1}, b_{2}$ are nonnegative constants. Obviously, $g$ satisfies conditions (1.2) and (1.6). Set

$$
G(s)=\int_{0}^{s} g(s) d s, \quad s \geq 0 .
$$


In order to state and prove our result, we introduce the following function space:

$$
\begin{aligned}
& Z=\left\{(u, v) \mid u, v \in L^{\infty}\left([0, T) ; W_{0}^{1,2(q+1)}(\Omega) \cap L^{2(p+2)}(\Omega)\right),\right. \\
& u_{t} \in L^{\infty}\left([0, T) ; L^{2}(\Omega)\right) \cap L^{m+1}(\Omega \times(0, T)), \\
& \left.v_{t} \in L^{\infty}\left([0, T) ; L^{2}(\Omega)\right) \cap L^{r+1}(\Omega \times(0, T)), u_{t t}, v_{t t} \in L^{\infty}\left([0, T), L^{2}(\Omega)\right)\right\} .
\end{aligned}
$$

Define the energy functional $E(t)$ associated with our system

$$
E(t)=\frac{1}{2}\left(\left\|u_{t}(t)\right\|_{2}^{2}+\left\|v_{t}(t)\right\|_{2}^{2}\right)+\frac{1}{2} \int_{\Omega}\left(G\left(|\nabla u|^{2}\right)+G\left(|\nabla v|^{2}\right)\right) d x-\int_{\Omega} F(u, v) d x
$$

A simple computation gives

$$
\frac{d E(t)}{d t}=-\|u\|_{m+1}^{m+1}-\|v\|_{r+1}^{r+1} \leq 0
$$

Our main result reads as follows.

Theorem 2.2. Assume that (2.3) holds. Assume further that $2(p+2)>\max \{2 q+2, m+1, r+1\}$. Then any solution of (1.1) with initial data satisfying

$$
\left(\int_{\Omega}\left(G\left(\left|\nabla u_{0}\right|^{2}\right)+G\left(\left|\nabla v_{0}\right|^{2}\right)\right) d x\right)^{1 / 2}>\alpha_{1}, \quad E(0)<E_{2}
$$

cannot exist for all time, where the constant $\alpha_{1}$ and $E_{2}$ are defined in (3.7).

\section{Proof of Theorem 2.2}

In this section, we deal with the blow-up of solutions of the system (1.1). Before we prove our main result, we need the following lemmas.

Lemma 3.1. Let $\Theta(t)$ be a solution of the ordinary differential inequality

$$
\frac{d \Theta(t)}{d t} \geq C \Theta^{1+\varepsilon}(t), \quad t>0,
$$

where $\varepsilon>0$. If $\Theta(0)>0$, then the solution ceases to exist for $t \geq \Theta^{-\varepsilon}(0) C^{-1} \varepsilon^{-1}$.

Lemma 3.2. Assume that (2.3) holds. Then there exists $\eta>0$ such that for any $(u, v) \in Z$, one has

$$
\|u+v\|_{2(p+2)}^{2(p+2)}+2\|u v\|_{p+2}^{p+2} \leq \eta\left(\int_{\Omega}\left(G\left(|\nabla u|^{2}\right)+G\left(|\nabla v|^{2}\right)\right) d x\right)^{p+2}
$$


Proof. By using Minkowski's inequality, we get

$$
\|u+v\|_{2(p+2)}^{2} \leq 2\left(\|u\|_{2(p+2)}^{2}+\|v\|_{2(p+2)}^{2}\right) .
$$

Also, Hölder's and Young's inequalities give us

$$
\|u v\|_{p+2} \leq\|u\|_{2(p+2)}\|v\|_{2(p+2)} \leq \frac{1}{2}\left(\|u\|_{2(p+2)}^{2}+\|v\|_{2(p+2)}^{2}\right) .
$$

If $b_{1}>0$, then we have

$$
\int_{\Omega}\left(G\left(|\nabla u|^{2}\right)+G\left(|\nabla v|^{2}\right)\right) d x \geq c\left(\|\nabla u\|_{2}^{2}+\|\nabla v\|_{2}^{2}\right) .
$$

If $b_{1}=0$, from $b_{1}+b_{2}>0$, we have $b_{2}>0$. Since $W_{0}^{1,2(q+1)}(\Omega) \hookrightarrow H_{0}^{1}(\Omega)$, we have

$$
\|\nabla u\|_{2}^{2}+\|\nabla v\|_{2}^{2} \leq c_{1}\left(\|\nabla u\|_{2(q+1)}^{2}+\|\nabla v\|_{2(q+1)}^{2}\right)
$$

which implies that (3.5) still holds for $b_{1}=0$. Combining (3.3), (3.4) with (3.5) and the embedding $H_{0}^{1}(\Omega) \hookrightarrow L^{2(p+2)}(\Omega)$, we have (3.2).

In order to prove our result and for the sake of simplicity, we take $a=b=1$ and introduce the following:

$$
\begin{gathered}
B=\eta^{1 /(2(p+2))}, \quad \alpha_{1}=B^{-(p+2 /(p+1))}, \quad E_{1}=\left(\frac{1}{2}-\frac{1}{2(p+2)}\right) \alpha_{1}^{2}, \\
E_{2}=\left(\frac{1}{2(q+1)}-\frac{1}{2(p+2)}\right) \alpha_{1}^{2}
\end{gathered}
$$

where $\eta$ is the optimal constant in (3.2). The following lemma will play an essential role in the proof of our main result, and it is similar to a lemma used first by Vitillaro [29].

Lemma 3.3. Assume that (2.3) holds. Let $(u, v) \in Z$ be the solution of the system (1.1). Assume further that $E(0)<E_{1}$ and

$$
\left(\int_{\Omega}\left(G\left(\left|\nabla u_{0}\right|^{2}\right)+G\left(\left|\nabla v_{0}\right|^{2}\right)\right) d x\right)^{1 / 2}>\alpha_{1}
$$

Then there exists a constant $\alpha_{2}>\alpha_{1}$ such that

$$
\begin{aligned}
& \left(\int_{\Omega}\left(G\left(|\nabla u|^{2}\right)+G\left(|\nabla v|^{2}\right)\right) d x\right)^{1 / 2} \geq \alpha_{2}, \quad \text { for } t>0 \\
& \left(\|u+v\|_{2(p+2)}^{2(p+2)}+2\|u v\|_{p+2}^{p+2}\right)^{1 /(2(p+2))} \geq B \alpha_{2}, \quad \text { for } t>0 .
\end{aligned}
$$


Proof. We first note that, by (2.10), (3.2), and the definition of $B$, we have

$$
\begin{aligned}
E(t) & \geq \frac{1}{2} \int_{\Omega}\left(G\left(|\nabla u|^{2}\right)+G\left(|\nabla v|^{2}\right)\right) d x-\frac{1}{2(p+2)}\left(\|u+v\|_{2(p+2)}^{2(p+2)}+2\|u v\|_{p+2}^{p+2}\right) \\
& \geq \frac{1}{2} \int_{\Omega}\left(G\left(|\nabla u|^{2}\right)+G\left(|\nabla v|^{2}\right)\right) d x-\frac{B^{2(p+2)}}{2(p+2)}\left(\int_{\Omega}\left(G\left(|\nabla u|^{2}\right)+G\left(|\nabla v|^{2}\right)\right) d x\right)^{p+2} \\
& =\frac{1}{2} \alpha^{2}-\frac{B^{2(p+2)}}{2(p+2)} \alpha^{2(p+2)},
\end{aligned}
$$

where $\alpha=\left(\int_{\Omega}\left(G\left(|\nabla u|^{2}\right)+G\left(|\nabla v|^{2}\right)\right) d x\right)^{1 / 2}$. It is not hard to verify that $g$ is increasing for $0<\alpha<\alpha_{1}$, decreasing for $\alpha>\alpha_{1}, g(\alpha) \rightarrow-\infty$ as $\alpha \rightarrow+\infty$, and

$$
g\left(\alpha_{1}\right)=\frac{1}{2} \alpha_{1}^{2}-\frac{B^{2(p+2)}}{2(p+2)} \alpha_{1}^{2(p+2)}=E_{1}
$$

where $\alpha_{1}$ is given in (3.7). Since $E(0)<E_{1}$, there exists $\alpha_{2}>\alpha_{1}$ such that $g\left(\alpha_{2}\right)=E(0)$.

Set $\alpha_{0}=\left(\int_{\Omega}\left(G\left(\left|\nabla u_{0}\right|^{2}\right)+G\left(\left|\nabla v_{0}\right|^{2}\right)\right) d x\right)^{1 / 2}$. Then by (3.11) we get $g\left(\alpha_{0}\right) \leq E(0)=g\left(\alpha_{2}\right)$, which implies that $\alpha_{0} \geq \alpha_{2}$. Now, to establish (3.9), we suppose by contradiction that

$$
\left(\int_{\Omega}\left(G\left(\left|\nabla u\left(t_{0}\right)\right|^{2}\right)+G\left(\left|\nabla v\left(t_{0}\right)\right|^{2}\right)\right) d x\right)^{1 / 2}<\alpha_{2}
$$

for some $t_{0}>0$. By the continuity of $\int_{\Omega}\left(G\left(|\nabla u|^{2}\right)+G\left(|\nabla v|^{2}\right)\right) d x$, we can choose $t_{0}$ such that

$$
\left(\int_{\Omega}\left(G\left(\left|\nabla u\left(t_{0}\right)\right|^{2}\right)+G\left(\left|\nabla v\left(t_{0}\right)\right|^{2}\right)\right) d x\right)^{1 / 2}>\alpha_{1}
$$

Again, the use of (3.11) leads to

$$
E\left(t_{0}\right) \geq g\left(\left(\int_{\Omega}\left(G\left(\left|\nabla u\left(t_{0}\right)\right|^{2}\right)+G\left(\left|\nabla v\left(t_{0}\right)\right|^{2}\right)\right) d x\right)^{1 / 2}\right)>g\left(\alpha_{2}\right)=E(0) .
$$

This is impossible since $E(t) \leq E(0)$ for all $t \in[0, T)$. Hence (3.9) is established.

To prove (3.10), we make use of (2.10) to get

$$
\frac{1}{2} \int_{\Omega}\left(G\left(|\nabla u|^{2}\right)+G\left(|\nabla v|^{2}\right)\right) d x \leq E(0)+\frac{1}{2(p+2)}\left(\|u+v\|_{2(p+2)}^{2(p+2)}+2\|u v\|_{p+2}^{p+2}\right)
$$


Consequently, (3.9) yields

$$
\begin{aligned}
\frac{1}{2(p+2)}\left(\|u+v\|_{2(p+2)}^{2(p+2)}+2\|u v\|_{p+2}^{p+2}\right) & \geq \frac{1}{2} \int_{\Omega}\left(G\left(|\nabla u|^{2}\right)+G\left(|\nabla v|^{2}\right)\right) d x-E(0) \\
& \geq \frac{1}{2} \alpha_{2}^{2}-E(0) \geq \frac{1}{2} \alpha_{2}^{2}-g\left(\alpha_{2}\right)=\frac{B^{2(p+2)}}{2(p+2)} \alpha_{2}^{2(p+2)} .
\end{aligned}
$$

Therefore, (3.17) and (3.7) yield the desired result.

Proof of Theorem 2.2. We suppose that the solution exists for all time and we reach to a contradiction. Set

$$
H(t)=E_{2}-E(t)
$$

By using (2.10) and (3.18), we have

$$
\begin{aligned}
0<H(0) \leq H(t)= & E_{2}-\frac{1}{2}\left(\left\|u_{t}(t)\right\|_{2}^{2}+\left\|v_{t}(t)\right\|_{2}^{2}\right)-\frac{1}{2} \int_{\Omega}\left(G\left(|\nabla u|^{2}\right)+G\left(|\nabla v|^{2}\right)\right) d x \\
& +\frac{1}{2(p+2)}\left(\|u+v\|_{2(p+2)}^{2(p+2)}+2\|u v\|_{p+2}^{p+2}\right) .
\end{aligned}
$$

From (3.9), we have

$$
\begin{gathered}
E_{2}-\frac{1}{2}\left(\left\|u_{t}(t)\right\|_{2}^{2}+\left\|v_{t}(t)\right\|_{2}^{2}\right)-\frac{1}{2} \int_{\Omega}\left(G\left(|\nabla u|^{2}\right)+G\left(|\nabla v|^{2}\right)\right) d x \\
\leq E_{2}-\frac{1}{2} \alpha_{1}^{2} \leq E_{1}-\frac{1}{2} \alpha_{1}^{2}=-\frac{1}{2(p+2)} \alpha_{1}^{2}<0, \quad \forall t \geq 0 .
\end{gathered}
$$

Hence, by the above inequality and (2.6), we have

$$
\begin{aligned}
0 & <H(0) \leq H(t) \leq \frac{1}{2(p+2)}\left(\|u+v\|_{2(p+2)}^{2(p+2)}+2\|u v\|_{p+2}^{p+2}\right), \\
& \leq \frac{c_{1}}{2(p+2)}\left(\|u\|_{2(p+2)}^{2(p+2)}+\|v\|_{2(p+2)}^{2(p+2)}\right) .
\end{aligned}
$$

We then define

$$
\Theta(t)=H^{1-\delta}(t)+\epsilon \int_{\Omega}\left(u u_{t}+v v_{t}\right) d x
$$

where $\epsilon$ small enough is to be chosen later and

$$
0<\delta \leq \min \left\{\frac{p+1}{2(p+2)}, \frac{2(p+2)-(m+1)}{2 m(p+2)}, \frac{2(p+2)-(r+1)}{2 r(p+2)}\right\}
$$


Our goal is to show that $\Theta(t)$ satisfies the differential inequality (3.1) which leads to a blowup in finite time. By taking a derivative of (3.23), we get

$$
\begin{aligned}
\Theta^{\prime}(t)= & (1-\delta) H^{-\delta}(t) H^{\prime}(t)+\epsilon\left(\left\|u_{t}\right\|_{2}^{2}+\left\|v_{t}\right\|_{2}^{2}\right)-\epsilon \int_{\Omega}\left(g\left(|\nabla u|^{2}\right)|\nabla u|^{2}+g\left(|\nabla v|^{2}\right)|\nabla v|^{2}\right) d x \\
& -\epsilon \int_{\Omega}\left(\left|u_{t}\right|^{m-1} u_{t} u+\left|v_{t}\right|^{r-1} v_{t} v\right) d x+\epsilon \int_{\Omega}\left(u f_{1}(u, v)+v f_{2}(u, v)\right) d x \\
= & (1-\delta) H^{-\delta}(t) H^{\prime}(t)+\epsilon\left(\left\|u_{t}\right\|_{2}^{2}+\left\|v_{t}\right\|_{2}^{2}\right)-b_{1} \epsilon\left(\|\nabla u\|_{2}^{2}+\|\nabla v\|_{2}^{2}\right)-\epsilon b_{2}\|\nabla u\|_{2(q+2)}^{2(q+2)} \\
& -\epsilon b_{2}\|\nabla v\|_{2(q+2)}^{2(q+2)}-\epsilon \int_{\Omega}\left(\left|u_{t}\right|^{m-1} u_{t} u+\left|v_{t}\right|^{r-1} v_{t} v\right) d x+\epsilon\left(\|u+v\|_{2(p+2)}^{2(p+2)}+2\|u v\|_{p+2}^{p+2}\right) .
\end{aligned}
$$

From the definition of $H(t)$, it follows that

$$
\begin{aligned}
-b_{2}\|\nabla u\|_{2(q+2)}^{2(q+2)}-b_{2}\|\nabla v\|_{2(q+2)}^{2(q+2)}= & 2(q+1) H(t)-2(q+1) E_{2}+(q+1)\left(\left\|u_{t}\right\|_{2}^{2}+\left\|v_{t}\right\|_{2}^{2}\right) \\
& +(q+1) b_{1}\left(\|\nabla u\|_{2}^{2}+\|\nabla v\|_{2}^{2}\right)-2(q+1) \int_{\Omega} F(u, v) d x,
\end{aligned}
$$

which together with (3.25) gives

$$
\begin{aligned}
\Theta^{\prime}(t)= & (1-\delta) H^{-\delta}(t) H^{\prime}(t)+\epsilon(q+2)\left(\left\|u_{t}\right\|_{2}^{2}+\left\|v_{t}\right\|_{2}^{2}\right)+b_{1} q \epsilon\left(\|\nabla u\|_{2}^{2}+\|\nabla v\|_{2}^{2}\right) \\
& -\epsilon \int_{\Omega}\left(\left|u_{t}\right|^{m-1} u_{t} u+\left|v_{t}\right|^{r-1} v_{t} v\right) d x+\epsilon\left(1-\frac{q+1}{p+2}\right)\left(\|u+v\|_{2(p+2)}^{2(p+2)}+2\|u v\|_{p+2}^{p+2}\right) \\
& +2(q+1) H(t)-2(q+1) E_{2} .
\end{aligned}
$$

Then, using (3.10), we obtain

$$
\begin{aligned}
\Theta^{\prime}(t) \geq & (1-\delta) H^{-\delta}(t) H^{\prime}(t)+\epsilon(q+2)\left(\left\|u_{t}\right\|_{2}^{2}+\left\|v_{t}\right\|_{2}^{2}\right)+b_{1} q \epsilon\left(\|\nabla u\|_{2}^{2}+\|\nabla v\|_{2}^{2}\right)+2(q+1) H(t) \\
& +\epsilon \bar{c}\left(\|u+v\|_{2(p+2)}^{2(p+2)}+2\|u v\|_{p+2}^{p+2}\right)-\epsilon \int_{\Omega}\left(\left|u_{t}\right|^{m-1} u_{t} u+\left|v_{t}\right|^{r-1} v_{t} v\right) d x,
\end{aligned}
$$

where $\bar{c}=1-(q+1) /(p+2)-2(q+1) E_{2}\left(B \alpha_{2}\right)^{-2(p+2)}$. It is clear that $\bar{c}>0$, since $\alpha_{2}>B^{-(p+2) /(p+1)}$. We now exploit Young's inequality to estimate the last two terms on the right side of (3.28)

$$
\begin{gathered}
\left.\left|\int_{\Omega}\right| u_{t}\right|^{m-1} u_{t} u d x \mid \leq \frac{\eta_{1}^{m+1}}{m+1}\|u\|_{m+1}^{m+1}+\frac{m \eta_{1}^{-((m+1) / m)}}{m+1}\left\|u_{t}\right\|_{m+1}^{m+1} \\
\left.\left|\int_{\Omega}\right| v_{t}\right|^{r-1} v_{t} v d x \mid \leq \frac{\eta_{2}^{r+1}}{r+1}\|v\|_{r+1}^{r+1}+\frac{r \eta_{2}^{-((r+1) / r)}}{r+1}\left\|v_{t}\right\|_{r+1}^{r+1},
\end{gathered}
$$


where $\eta_{1}, \eta_{2}$ are parameters depending on the time $t$ and specified later. Inserting the last two estimates into (3.28), we have

$$
\begin{aligned}
\Theta^{\prime}(t) \geq & (1-\delta) H^{-\delta}(t) H^{\prime}(t)+\epsilon(q+2)\left(\left\|u_{t}\right\|_{2}^{2}+\left\|v_{t}\right\|_{2}^{2}\right)+b_{1} q \epsilon\left(\|\nabla u\|_{2}^{2}+\|\nabla v\|_{2}^{2}\right)+2(q+1) H(t) \\
& +\epsilon \bar{c}\left(\|u+v\|_{2(p+2)}^{2(p+2)}+2\|u v\|_{p+2}^{p+2}\right)-\epsilon \frac{\eta_{1}^{m+1}}{m+1}\|u\|_{m+1}^{m+1}-\epsilon \frac{m \eta_{1}^{-((m+1) / m)}}{m+1}\left\|u_{t}\right\|_{m+1}^{m+1} \\
& -\epsilon \frac{\eta_{2}^{r+1}}{r+1}\|v\|_{r+1}^{r+1}-\epsilon \frac{r \eta_{2}^{-((r+1) / r)}}{r+1}\left\|v_{t}\right\|_{r+1}^{r+1} .
\end{aligned}
$$

By choosing $\eta_{1}$ and $\eta_{2}$ such that

$$
\eta_{1}^{-(m+1) / m}=M_{1} H^{-\delta}(t), \quad \eta_{2}^{-(r+1) / r}=M_{2} H^{-\delta}(t),
$$

where $M_{1}$ and $M_{2}$ are constants to be fixed later. Thus, by using (2.6) and (3.31), inequality (3.31) then takes the form

$$
\begin{aligned}
\Theta^{\prime}(t) \geq & ((1-\delta)-M \epsilon) H^{-\delta}(t) H^{\prime}(t)+\epsilon(q+2)\left(\left\|u_{t}\right\|_{2}^{2}+\left\|v_{t}\right\|_{2}^{2}\right)+b_{1} q \epsilon\left(\|\nabla u\|_{2}^{2}+\|\nabla v\|_{2}^{2}\right) \\
& +2(q+1) H(t)+\epsilon c_{2}\left(\|u\|_{2(p+2)}^{2(p+2)}+2\|v\|_{2(p+2)}^{2(p+2)}\right)-\epsilon M_{1}^{-m} H^{\delta m}(t)\|u\|_{m+1}^{m+1} \\
& -\epsilon M_{2}^{-r} H^{\delta r}(t)\|v\|_{r+1}^{r+1}
\end{aligned}
$$

where $M=m /(m+1) M_{1}+r /(r+1) M_{2}$ and $c_{2}$ is a positive constant.

Since $2(p+2)>\max \{m+1, r+1\}$, taking into account (2.6) and (3.21), then we have

$$
\begin{gathered}
H^{\delta m}(t)\|u\|_{m+1}^{m+1} \leq c_{3}\left(\|u\|_{2(p+2)}^{2 \delta m(p+2)+(m+1)}+\|v\|_{2(p+2)}^{2 \delta m(p+2)}\|u\|_{m+1}^{m+1}\right), \\
H^{\delta r}(t)\|v\|_{r+1}^{r+1} \leq c_{4}\left(\|v\|_{2(p+2)}^{2 \delta r(p+2)+(r+1)}+\|u\|_{2(p+2)}^{2 \delta r(p+2)}\|v\|_{r+1}^{r+1}\right),
\end{gathered}
$$

for some positive constants $c_{3}$ and $c_{4}$. By using (3.24) and the algebraic inequality

$$
z^{v} \leq z+1 \leq\left(1+\frac{1}{a}\right)(z+a), \quad \forall z \geq 0,0<v \leq 1, a \geq 0
$$

we have

$$
\|u\|_{2(p+2)}^{2 \delta m(p+2)+(m+1)} \leq d\left(\|u\|_{2(p+2)}^{2(p+2)}+H(0)\right) \leq d\left(\|u\|_{2(p+2)}^{2(p+2)}+H(t)\right), \quad \forall t \geq 0,
$$


where $d=1+1 / H(0)$. Similarly,

$$
\|v\|_{2(p+2)}^{2 \delta r(p+2)+(r+1)} \leq d\left(\|v\|_{2(p+2)}^{2(p+2)}+H(t)\right), \quad \forall t \geq 0 .
$$

Also, since

$$
(X+Y)^{s} \leq C\left(X^{s}+Y^{s}\right), \quad X, Y \geq 0, s>0,
$$

by using (3.24) and (3.34), we conclude that

$$
\begin{gathered}
\|v\|_{2(p+2)}^{2 \delta m(p+2)}\|u\|_{m+1}^{m+1} \leq C\left(\|v\|_{2(p+2)}^{2(p+2)}+\|u\|_{(m+1)}^{2(p+2)}\right) \leq C\left(\|v\|_{2(p+2)}^{2(p+2)}+\|u\|_{2(p+2)}^{2(p+2)}\right), \\
\|u\|_{2(p+2)}^{2 \delta r(p+2)}\|v\|_{r+1}^{r+1} \leq C\left(\|u\|_{2(p+2)}^{2(p+2)}+\|v\|_{(r+1)}^{2(p+2)}\right) \leq C\left(\|u\|_{2(p+2)}^{2(p+2)}+\|v\|_{2(p+2)}^{2(p+2)}\right),
\end{gathered}
$$

where $C$ is a generic positive constant. Taking into account (3.33)-(3.38), estimate (3.32) takes the form

$$
\begin{aligned}
\Theta^{\prime}(t) \geq & ((1-\delta)-M \epsilon) H^{-\delta}(t) H^{\prime}(t)+\epsilon(q+2)\left(\left\|u_{t}\right\|_{2}^{2}+\left\|v_{t}\right\|_{2}^{2}\right) \\
& +\epsilon\left(2(q+1)-C_{1} M_{1}^{-m}-C_{1} M_{2}^{-r}\right) H(t) \\
& +\epsilon\left(c_{2}-C_{2} M_{1}^{-m}-C_{2} M_{2}^{-r}\right)\left(\|u\|_{2(p+2)}^{2(p+2)}+\|v\|_{2(p+2)}^{2(p+2)}\right)
\end{aligned}
$$

where $C_{1}=\max \left\{c_{3} d+C, c_{4} d+C\right\}, C_{2}=\max \left\{c_{3} d, c_{4} d\right\}$. At this point, and for large values of $M_{1}$ and $M_{2}$, we can find positive constants $\kappa_{1}$ and $\kappa_{2}$ such that (3.39) becomes

$$
\begin{aligned}
\Theta^{\prime}(t) \geq & ((1-\delta)-M \epsilon) H^{-\delta}(t) H^{\prime}(t)+\epsilon(q+2)\left(\left\|u_{t}\right\|_{2}^{2}+\left\|v_{t}\right\|_{2}^{2}\right) \\
& +\epsilon \mathcal{K}_{1} H(t)+\epsilon \mathcal{K}_{2}\left(\|u\|_{2(p+2)}^{2(p+2)}+\|v\|_{2(p+2)}^{2(p+2)}\right) .
\end{aligned}
$$

Once $M_{1}$ and $M_{2}$ are fixed, we pick $\epsilon$ small enough so that $(1-\delta)-M \epsilon \geq 0$ and

$$
\Theta(0)=H^{1-\delta}(0)+\epsilon \int_{\Omega}\left(u_{0} u_{1}+v_{0} v_{1}\right) d x>0
$$

Since $H^{\prime}(t) \geq 0$, there exists $\Lambda>0$ such that (3.40) becomes

$$
\Theta^{\prime}(t) \geq \epsilon \Lambda\left(H(t)+\left\|u_{t}\right\|_{2}^{2}+\left\|v_{t}\right\|_{2}^{2}+\|u\|_{2(p+2)}^{2(p+2)}+\|v\|_{2(p+2)}^{2(p+2)}\right) .
$$

Then, we have

$$
\Theta(t) \geq \Theta(0), \quad \forall t \geq 0 .
$$


Next, we have by Hölder's and Young's inequalities

$$
\left(\int_{\Omega} u u_{t} d x+\int_{\Omega} v v_{t} d x\right)^{1 /(1-\delta)} \leq C\left(\|u\|_{2(p+2)}^{\tau /(1-\delta)}+\left\|u_{t}\right\|_{2}^{S /(1-\delta)}+\|v\|_{2(p+2)}^{\tau /(1-\delta)}+\left\|v_{t}\right\|_{2}^{\delta /(1-\delta)}\right)
$$

for $1 / \tau+1 / s=1$. We take $s=2(1-\delta)$, to get $\tau /(1-\delta)=2 /(1-2 \delta)$. Here and in the sequel, $C$ denotes a positive constant which may change from line to line. By using (3.24) and (3.34), we have

$$
\|u\|_{2(p+2)}^{2 /(1-2 \delta)} \leq d\left(\|u\|_{2(p+2)}^{2(p+2)}+H(t)\right), \quad\|v\|_{2(p+2)}^{2 /(1-2 \delta)} \leq d\left(\|v\|_{2(p+2)}^{2(p+2)}+H(t)\right), \quad \forall t \geq 0 .
$$

Therefore, (3.44) becomes

$$
\left(\int_{\Omega} u u_{t} d x+\int_{\Omega} v v_{t} d x\right)^{1 /(1-\delta)} \leq C\left(\|u\|_{2(p+2)}^{2(p+2)}+\|v\|_{2(p+2)}^{2(p+2)}+\left\|u_{t}\right\|_{2}^{2}+\left\|v_{t}\right\|_{2}^{2}\right)
$$

Note that

$$
\begin{aligned}
\Theta^{1 /(1-\delta)}(t) & =\left(H^{1-\delta}(t)+\epsilon \int_{\Omega}\left(u u_{t}+v v_{t}\right) d x\right)^{1 /(1-\delta)} \\
& \leq C\left(H(t)+\left|\int_{\Omega} u u_{t} d x+\int_{\Omega} v v_{t} d x\right|^{1 /(1-\delta)}\right) \\
& \leq C\left(H(t)+\|u\|_{2(p+2)}^{2(p+2)}+\|v\|_{2(p+2)}^{2(p+2)}+\left\|u_{t}\right\|_{2}^{2}+\left\|v_{t}\right\|_{2}^{2}\right) .
\end{aligned}
$$

Combining (3.42) with (3.47), we have

$$
\Theta(t) \geq C \Theta^{1 /(1-\delta)}(t), \quad \forall t \geq 0
$$

A simple application of Lemma 3.1 gives the desired result.

\section{Acknowledgments}

The authors are indebted to the referee for giving some important suggestions which improved the presentations of this paper. This work is supported in part by a China NSF Grant no. 10871097, Qing Lan Project of Jiangsu Province, the Foundation for Young Talents in College of Anhui Province Grant no. 2011SQRL115 and Program sponsored for scientific innovation research of college graduate in Jangsu province. 


\section{References}

[1] A. Haraux and E. Zuazua, "Decay estimates for some semilinear damped hyperbolic problems," Archive for Rational Mechanics and Analysis, vol. 100, no. 2, pp. 191-206, 1988.

[2] J. M. Ball, "Remarks on blow-up and nonexistence theorems for nonlinear evolution equations," The Quarterly Journal of Mathematics. Oxford. Second Series, vol. 28, no. 112, pp. 473-486, 1977.

[3] V. K. Kalantarov, "The occurrence of collapse for quasilinear equations of parabolic and hyperbolic types," Journal of Soviet Mathematics, vol. 10, no. 1, pp. 53-70, 1978.

[4] H. A. Levine, "Instability and nonexistence of global solutions to nonlinear wave equations of the form $P u_{t t}=-A u+\mathcal{F}(u), "$ Transactions of the American Mathematical Society, vol. 192, pp. 1-21, 1974.

[5] H. A. Levine, "Some additional remarks on the nonexistence of global solutions to nonlinear wave equations," SIAM Journal on Mathematical Analysis, vol. 5, pp. 138-146, 1974.

[6] V. Georgiev and G. Todorova, "Existence of a solution of the wave equation with nonlinear damping and source terms," Journal of Differential Equations, vol. 109, no. 2, pp. 295-308, 1994.

[7] S. A. Messaoudi, "Blow up in a nonlinearly damped wave equation," Mathematische Nachrichten, vol. 231, pp. 105-111, 2001.

[8] S. A. Messaoudi, "Blow-up of positive-initial-energy solutions of a nonlinear viscoelastic hyperbolic equation," Journal of Mathematical Analysis and Applications, vol. 320, no. 2, pp. 902-915, 2006.

[9] G. Andrews, "On the existence of solutions to the equation $u_{t t}=u_{x x t}+\sigma\left(u_{x}\right)_{x}$," Journal of Differential Equations, vol. 35, no. 2, pp. 200-231, 1980.

[10] G. Andrews and J. M. Ball, "Asymptotic behaviour and changes of phase in one-dimensional nonlinear viscoelasticity," Journal of Differential Equations, vol. 44, no. 2, pp. 306-341, 1982.

[11] J. Clements, "Existence theorems for a quasilinear evolution equation," SIAM Journal on Applied Mathematics, vol. 26, pp. 745-752, 1974.

[12] S. Kawashima and Y. Shibata, "Global existence and exponential stability of small solutions to nonlinear viscoelasticity," Communications in Mathematical Physics, vol. 148, no. 1, pp. 189-208, 1992.

[13] D. D Ang and A. P. N. Dinh, "Strong solutions of a quasilinear wave equation with nonlinear damping," SIAM Journal on Mathematical Analysis, vol. 19, no. 2, pp. 337-347, 1988.

[14] W. N. Findley, J. S. Lai, and K. Onaran, Creep and Relaxation of Nonlinear Viscoelastic Materials, NorthHolland, Amsterdam, The Netherlands, 1976.

[15] J. K. Knowles, "On finite anti-plane shear for incompressible elastic materials," Australian Mathematical Society. Journal. Series B, vol. 19, no. 4, pp. 400-415, 1975/76.

[16] V. P. Maslov and P. P. Mosolov, Nonlinear Wave Equations Perturbed by Viscous Terms, vol. 31 of de Gruyter Expositions in Mathematics, Walter De Gruyter, Berlin, Germany, 2000.

[17] J. M. Greenberg, R. C. MacCamy, and V. J. Mizel, "On the existence, uniqueness, and stability of solutions of the equation $\sigma^{\prime}\left(u_{x}\right) u_{x x}+\lambda u_{x x t}=\rho_{0} u_{t t}, "$ vol. 17, pp. 707-728, 1967/1968.

[18] Y. Yamada, "Some remarks on the equation $Y_{t t}-\sigma\left(Y_{x}\right) Y_{x x}-Y_{x x t}=f$," Osaka Journal of Mathematics, vol. 17, no. 2, pp. 303-323, 1980.

[19] Z. Yang and G. Chen, "Global existence of solutions for quasi-linear wave equations with viscous damping," Journal of Mathematical Analysis and Applications, vol. 285, no. 2, pp. 604-618, 2003.

[20] G. Chen, H. Yue, and S. Wang, "The initial boundary value problem for quasi-linear wave equation with viscous damping," Journal of Mathematical Analysis and Applications, vol. 331, no. 2, pp. 823-839, 2007.

[21] J. Hao, Y. Zhang, and S. Li, "Global existence and blow-up phenomena for a nonlinear wave equation," Nonlinear Analysis, vol. 71, no. 10, pp. 4823-4832, 2009.

[22] S. A. Messaoudi and B. Said Houari, "Global non-existence of solutions of a class of wave equations with non-linear damping and source terms," Mathematical Methods in the Applied Sciences, vol. 27, no. 14, pp. 1687-1696, 2004.

[23] I. E. Segal, "Nonlinear partial differential equations in quantum field theory," in Proc. Sympos. Appl. Math., Vol. XVII, pp. 210-226, American Mathematical Society, Providence, RI, USA, 1965.

[24] K. Jorgens, Nonlinear Wave Equations, University of Colorado, Department of Mathematics, 1970.

[25] K. Agre and M. A. Rammaha, "Systems of nonlinear wave equations with damping and source terms," Differential and Integral Equations, vol. 19, no. 11, pp. 1235-1270, 2006.

[26] B. Said-Houari, "Global nonexistence of positive initial-energy solutions of a system of nonlinear wave equations with damping and source terms," Differential and Integral Equations, vol. 23, no. 1-2, pp. 79-92, 2010.

[27] J. Wu, S. Li, and S. Chai, "Existence and nonexistence of a global solution for coupled nonlinear wave equations with damping and source," Nonlinear Analysis, vol. 72, no. 11, pp. 3969-3975, 2010. 
[28] L. E. Payne and D. H. Sattinger, "Saddle points and instability of nonlinear hyperbolic equations," Israel Journal of Mathematics, vol. 22, no. 3-4, pp. 273-303, 1975.

[29] E. Vitillaro, "Global nonexistence theorems for a class of evolution equations with dissipation," Archive for Rational Mechanics and Analysis, vol. 149, no. 2, pp. 155-182, 1999.

[30] S. A. Messaoudi and B. Said-Houari, "Global nonexistence of positive initial-energy solutions of a system of nonlinear viscoelastic wave equations with damping and source terms," Journal of Mathematical Analysis and Applications, vol. 365, no. 1, pp. 277-287, 2010. 


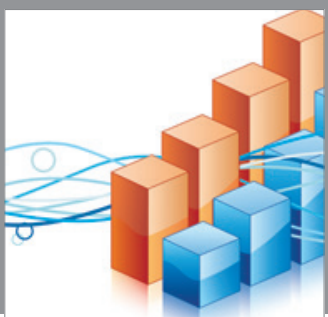

Advances in

Operations Research

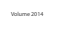

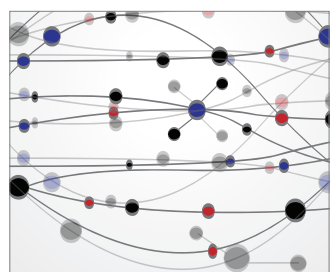

\section{The Scientific} World Journal
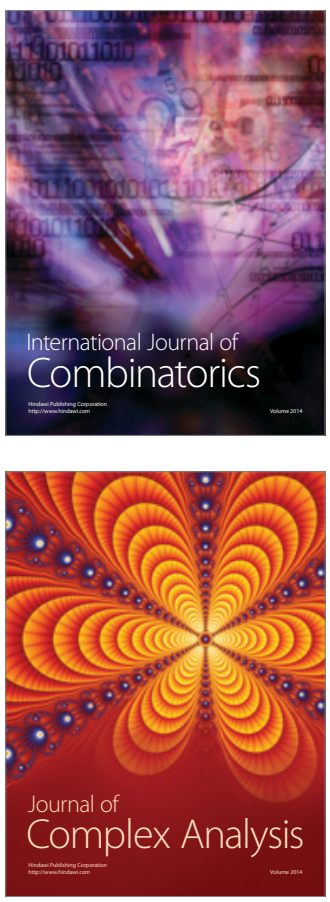

International Journal of

Mathematics and

Mathematical

Sciences
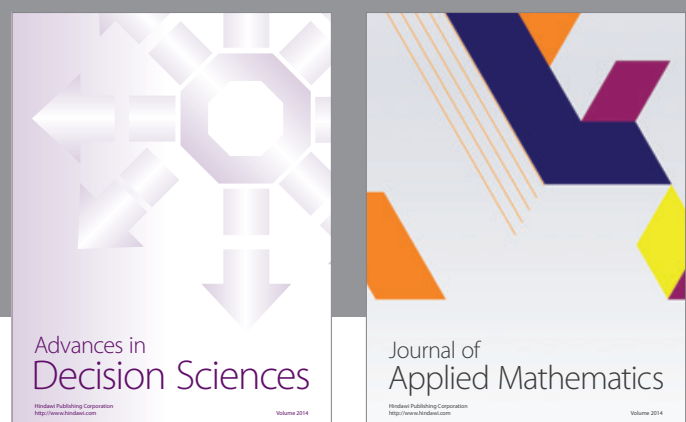

Journal of

Applied Mathematics
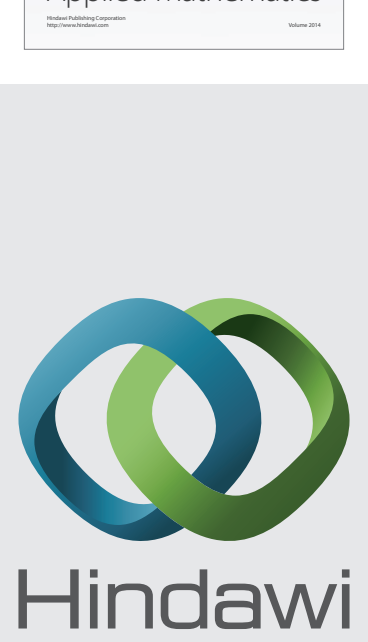

Submit your manuscripts at http://www.hindawi.com
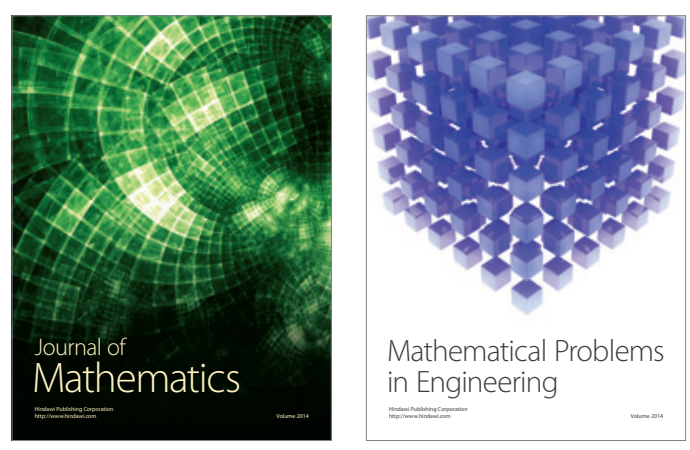

Mathematical Problems in Engineering
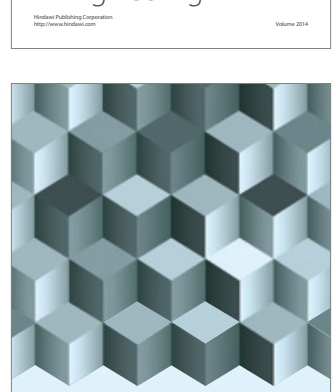

Journal of

Function Spaces
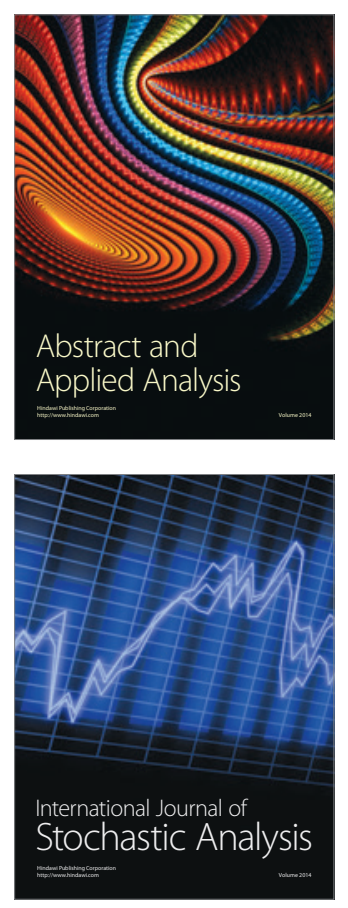

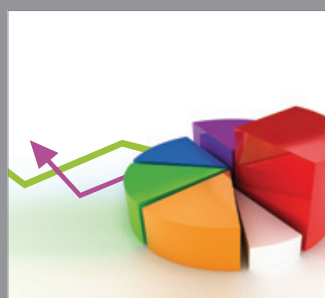

ournal of

Probability and Statistics

Promensencen
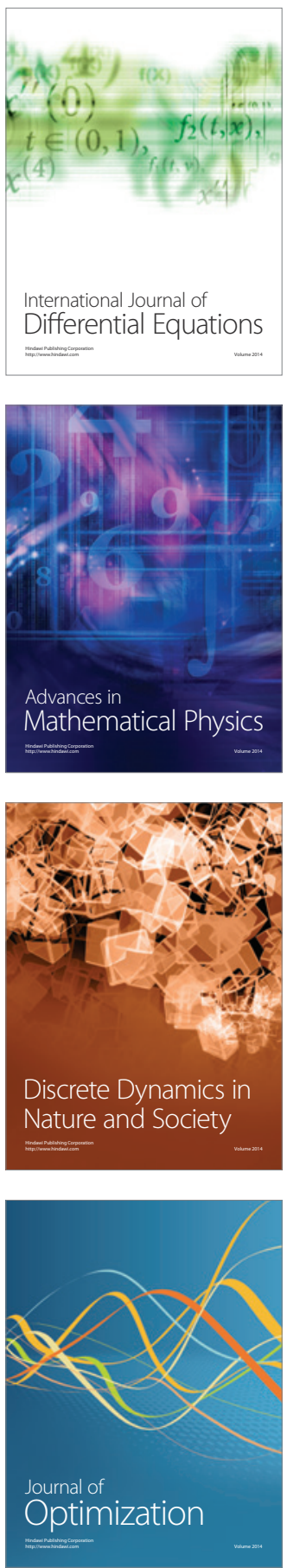